\title{
Effect of partitioning of monomer and emulsifier in aqueous media on particle formation in emulsion homopolymerization of hydrophobic and hydrophilic monomers with a nonionic emulsifier ${ }^{\dagger}$
}

\begin{abstract}
Nami Matsusaka ${ }^{1}$, Toyoko Suzuki ${ }^{1}$ and Masayoshi Okubo ${ }^{1,2}$
Styrene (hydrophobic monomer), methyl methacrylate (MMA, hydrophilic monomer, almost 100 times more soluble in water than styrene) and nonionic emulsifier are often used in general emulsion polymerizations in industrial production. To clarify the effect of stirring for $100 \mathrm{~min}$ at $70^{\circ} \mathrm{C}$ before starting emulsion polymerization with a nonionic emulsifier (polyoxyethylene nonyl phenyl ether, E911) and potassium persulfate as the initiator for the particle formation, the emulsion homopolymerization of styrene and MMA was carried out at different stirring rates $\left(0-500\right.$ r.p.m.) at $70{ }^{\circ} \mathrm{C}$. Immediately before the start of the polymerization, in the case of styrene, partitioning of components in the system nearly reached an equilibrium state at 500 r.p.m., whereas the system was in a nonequilibrium state at 240 r.p.m. In the case of MMA, the system nearly reached an equilibrium state even at 240 r.p.m. Polystyrene particles were obtained as $\sim 100$-nm-sized spherical particles at 240 r.p.m. and as $\sim 1-\mu \mathrm{m}$-sized 'bur-like' nonspherical particles at 500 r.p.m., whereas poly(MMA) particles were $\sim 100-\mathrm{nm}$-sized and almost spherical at both stirring rates. Monomer polarity strongly affected the partitioning of the monomer to the aqueous phase and the nonionic emulsifier to the monomer phase before starting the emulsion polymerization, resulting in the differences in particle formation and the incorporation of nonionic emulsifier inside the polymer particles.
\end{abstract}

Polymer Journal (2013) 45, 153-159; doi:10.1038/pj.2012.109; published online 20 June 2012

Keywords: emulsion polymerization; incorporation; monomer polarity; nonionic emulsifier; nonspherical particle; partitioning

\section{INTRODUCTION}

Nonionic emulsifiers have been used in the preparation of polymer colloids by emulsion polymerization. Nonionic emulsifiers offer various advantages compared with ionic emulsifiers, such as higher chemical and freeze-thaw stability, higher pigment affinity and less effervescence of the emulsion. In the emulsion polymerization with nonionic emulsifier, nonionic emulsifier has been reported to move from an aqueous medium into a monomer phase before starting the polymerization. ${ }^{1-7}$ When monomer droplets (layer) disappear during emulsion polymerization, the emulsifiers inside are instantly released to the aqueous phase, resulting in a higher concentration in the aqueous phase than the critical micelle concentration $(\mathrm{cmc}) .^{2-5}$ We found that quantities of nonionic emulsifier released from monomer droplets entered polymerizing particles swollen with monomer via the aqueous phase and remained within these particles until the end of polymerization, ${ }^{8-13}$ as described below.

In the emulsion copolymerization of styrene and methacrylic acid (MAA) (MAA, $10 \mathrm{~mol} \%$ ) with the nonionic emulsifier polyoxyethylene nonyl phenyl ether (Emulgen 911 (E911), $\mathrm{C}_{9} \mathrm{H}_{19^{-}}$ $\mathrm{C}_{6} \mathrm{H}_{4}-\mathrm{O}\left(\mathrm{CH}_{2} \mathrm{CH}_{2} \mathrm{O}\right)_{10.9} \mathrm{H}$, hydrophilic-lipophilic balance (HLB) $13.7),{ }^{8} 75 \%$ of the emulsifier was incorporated inside the copolymer particles.

We recently proposed a mechanism for this incorporation of nonionic emulsifier inside particles in emulsion polymerization. Most nonionic emulsifiers in the aqueous phase are dissolved in the monomer droplets (layer) because of the high affinity between emulsifier and monomer in the early stages of the polymerization. Throughout the polymerization, the emulsifiers diffuse continuously into monomerswollen particles through the aqueous medium from monomer droplets (layers) until the monomer phase disappears because the monomer is supplied to the primary particles, and the amount of nonionic emulsifier dissolved in the monomer droplets (layers) is decreased. As a result, a significant amount of emulsifier would be located inside the polymer particles at the completion of the polymerization.

Such an incorporation of nonionic emulsifier inside particles was also observed in the emulsion polymerization of styrene using two 
kinds of nonionic emulsifiers with different HLB values. The two types of nonionic emulsifiers are Emulgen 109P (E109P) $\left[\mathrm{C}_{12} \mathrm{H}_{25} \mathrm{O}\left(\mathrm{CH}_{2} \mathrm{CH}_{2} \mathrm{O}\right)_{9.2} \mathrm{H}\right.$, HLB 13.6] and Emulgen $150(\mathrm{E} 150)\left[\mathrm{C}_{12}\right.$ $\mathrm{H}_{25} \mathrm{O}\left(\mathrm{CH}_{2} \mathrm{CH}_{2} \mathrm{O}\right)_{47.4} \mathrm{H}$, HLB 18.3]. ${ }^{9}$ In the emulsion polymerization of methyl methacrylate, ethyl methacrylate or isobutyl methacrylate using E109P or E911, ${ }^{10}$ the percentage of incorporation depended on the kinds of nonionic emulsifier and monomer. In addition, we previously explained the effects of stirring on the partitioning of monomer and emulsifier before starting the emulsion polymerization of styrene. ${ }^{14}$

In this article, the effect of stirring upon particle formation with nonionic emulsifier before starting the emulsion homopolymerization of styrene and methyl methacrylate (MMA), which are typical hydrophobic and hydrophilic monomers, will be clarified.

\section{EXPERIMENTAL PROCEDURE}

\section{Materials}

Styrene (Mitsubishi Chemical Co, Tokyo, Japan) and MMA (Nacalai Tesque Inc., Tokyo, Japan) were purified by distillation under reduced pressure in a nitrogen atmosphere. Analytical grade potassium persulfate (KPS, Nacalai Tesque Inc.) was purified by recrystallization. Commercial grade nonionic polyoxyethylene nonyl phenyl ether nonionic emulsifier (Emulgen 911 (E911), HLB 13.7, Kao Co., Tokyo, Japan) with an average of 10.9 ethylene oxides per molecule, and guaranteed reagent grade 2-propanol and tetrahydrofuran (Nacalai Tesque Inc.) were used as received. Water used in all experiments was obtained from an Elix UV (Millipore Co., Ltd., Tokyo, Japan) purification system and had a resistivity of $18.2 \mathrm{M} \Omega \mathrm{cm}^{-1}$.

\section{Emulsion polymerization}

Polystyrene (PS) and poly(MMA) (PMMA) particles were prepared by emulsion polymerization with E911 using a reaction calorimeter (RC-1e; Mettler-Toledo, Tokyo, Japan) with a 100-ml cylinder type reactor under the conditions shown in Table 1. A three-blade stirrer was set $1.5 \mathrm{~cm}$ from the bottom of the RC-le reactor and $4 \mathrm{~cm}$ from the interface between the monomer and water layers without stirring. At a stirring rate of 240 r.p.m. (referred to as 'inefficient' stirring), most of the monomer was floating on an aqueous (water and micelles) phase. At a stirring rate of 500 r.p.m. (referred to as 'efficient' stirring), most of the monomer was dispersed as droplets in the aqueous phase. E911 aqueous solution was charged into the reactor and purged with nitrogen for $15 \mathrm{~min}$. After the addition of the monomer to the reactor, the internal temperature was raised in a linear manner from room temperature to $\sim 70^{\circ} \mathrm{C}$ over a period of $20 \mathrm{~min}$ and then kept at $70^{\circ} \mathrm{C}$ for $80 \mathrm{~min}$ with stirring as shown in Figure 1. For a total of $100 \mathrm{~min}$, this process was carried out to provide temperature calibration of the reaction calorimeter. Emulsion polymerizations were started by the addition of KPS aqueous solution. In all cases, the final conversions as measured by gravimetry were over $90 \%$.

Concentrations of E911 and monomer in the aqueous phase just before starting emulsion polymerization

The same mixture as in the above emulsion polymerization was used except that the initiator was stirred in the $\mathrm{RC}-1 \mathrm{e}$ reactor at various rates (0-500 r.p.m.) for $100 \mathrm{~min}$ using the temperature profile shown in Figure 1. Stopping the stirring for $\sim 5 \mathrm{~min}$ created a two-layer separation of the aqueous and monomer phases; then, part of the lower aqueous layer was taken out from the bottom of the reactor. The sample of the lower aqueous layer was centrifuged at $2800 \mathrm{~g}$ for $5 \mathrm{~min}$ to remove the monomer droplets completely, and the quantities of E911 and monomers in the aqueous phase were determined by gravimetry and gas chromatography.

\section{Characterization of PS and PMMA particles}

PS and PMMA particles were observed with a transmission electron microscope (TEM, JEM-1230, JEOL Ltd, Tokyo, Japan). A drop of each emulsion was placed onto a carbon-coated copper grid and allowed to dry in a desiccator at room temperature.
Table 1 Recipe for the preparation of PS and PMMA particles by emulsion polymerization ${ }^{a}$ with nonionic emulsifier at various stirring rates in a RC-1e reactor

Ingredients

\begin{tabular}{lcc}
\hline Monomer $^{\mathrm{b}}$ & $2.0 \mathrm{~g}$ & \\
KPS $^{\mathrm{c}}$ & $0.16 \mathrm{~g}$ & $\left(8045 \mathrm{mmoll}^{-1}\right.$-water $)$ \\
Emulgen 911 & $0.20 \mathrm{~g}$ & \\
Water & $70 \mathrm{~g}$ & $\left(4.14 \mathrm{mmoll}^{-1}\right.$-water $)$
\end{tabular}

Abbreviations: KPS, potassium persulfate; PMMA, poly methacrylic acid; PS, Polystyrene ${ }^{a} \mathrm{~N}_{2} ; 70{ }^{\circ} \mathrm{C} ; 6 \mathrm{~h}$; various stirring rates $(0-500$ r.p.m.). Styrene or MMA.

cAdded as aqueous solution $(10 \mathrm{ml})$ after $100 \mathrm{~min}$.
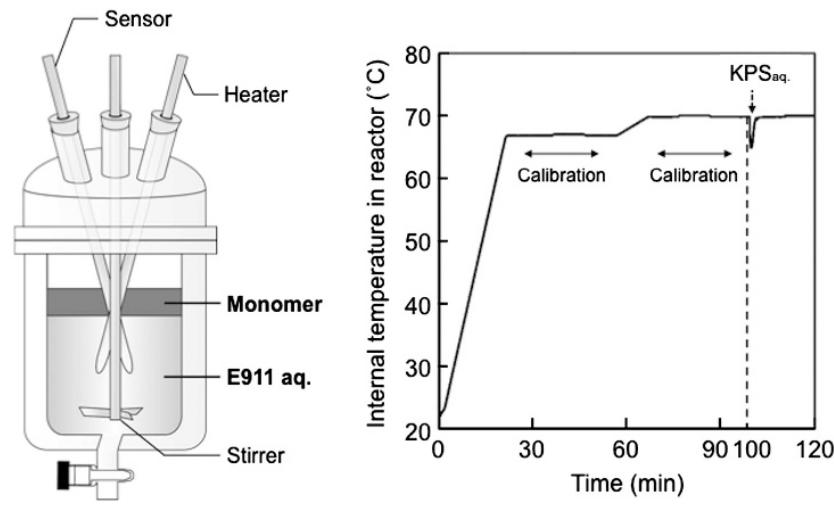

Figure 1 Profiles of the steady state (right) and of reactor temperature before starting emulsion polymerization as a function of time for the initial calibration with reaction calorimeter $\mathrm{RC}-1 \mathrm{e}$ (left).

Number-average diameters $\left(d_{\mathrm{n}}\right)$ of free and monomer-solubilized micelles were measured by a super-dynamic light scattering spectrophotometer (DLS 7000 ( $\mathrm{Ar}^{+}$laser) and FPAR-1000RK, Otsuka Electronics Co. Ltd, Osaka, Japan). The measurements were carried out at $25^{\circ} \mathrm{C}$ at a scattering angle of $30^{\circ}$.

The amount of E911 incorporated inside the polymer particles was analyzed by GPC using two S/divinylbenzene gel columns (Tosoh Corporation, Yamaguchi, Japan, TSKgel $\mathrm{GMH}_{\mathrm{HR}^{-}} \mathrm{H}, 7.8 \mathrm{~mm}$ i.d. $\times 30 \mathrm{~cm}$; separation range per column: $\sim 50-4 \times 10^{8} \mathrm{~g} \mathrm{~mol}^{-1}$ (exclusion limit)). Following the protocol described in our previous article, the PS and PMMA particles were centrifugally washed three times with 2-propanol and $20 \mathrm{wt} \%$ 2-propanol aqueous solution to completely remove the E911 in the aqueous phase and on the particle surfaces, and the PS and PMMA particles were dissolved in tetrahydrofuran. ${ }^{13}$

\section{RESULTS AND DISCUSSION}

E911 concentration in the aqueous phase just before starting emulsion polymerization of styrene and MMA

Emulsion homopolymerization of styrene and MMA with nonionic emulsifier E911 was carried out at various stirring rates (0-500 r.p.m.). In this article, the sample names and 'monomerstirring rate' have been abbreviated: for example, S-500 means 'emulsion polymerization of styrene (S) with stirring at 500 r.p.m.'

Figure 2 shows E911 concentrations in the aqueous media (water and micelles) ([E911 $]_{\mathrm{aq}}$.) just before starting the emulsion polymerizations of styrene (a) and MMA (b) at $3 \mathrm{wt} \%$ monomer concentration as a function of the stirring rate. In the case of styrene, $[\mathrm{E} 911]_{\mathrm{aq}}$. decreased, indicating that E911 transferred from the aqueous medium to the monomer phase with an increase in the stirring rate. In the case of MMA, [E911 $]_{\mathrm{aq}}$. values at various stirring rates were almost constant at a high value, indicating that almost all E911 remained in the aqueous medium. These results should be based on the 

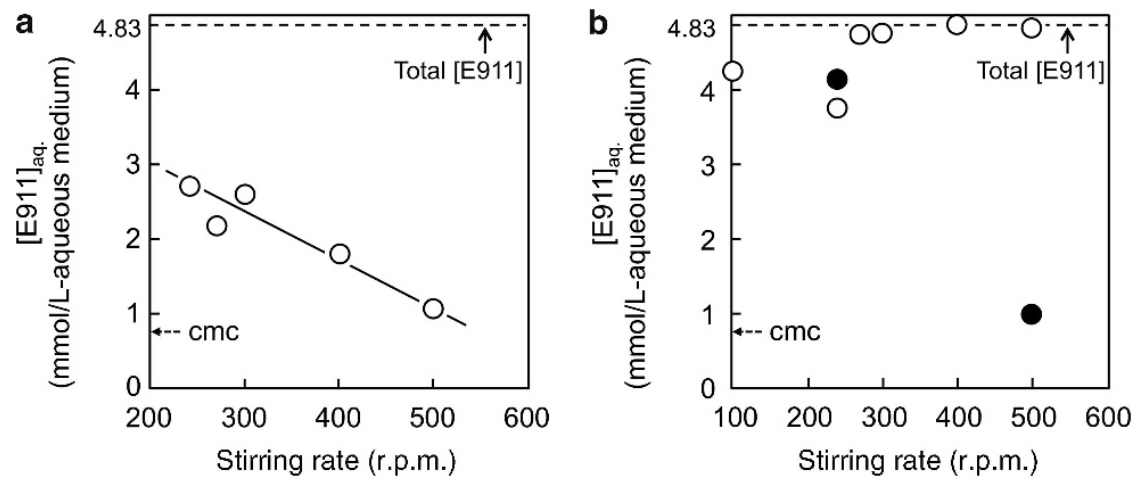

Figure 2 E911 concentration in the aqueous phase (water and micelles) after stirring at various rates for $100 \mathrm{~min} \sim 70^{\circ} \mathrm{C}$ before starting the emulsion polymerization: monomer (styrene (a) or MMA (b)) $2.0 \mathrm{~g}$; E911 $0.20 \mathrm{~g}$; water $60 \mathrm{~g}$. Initial [E911]aq. (mmol I-1-water) $=4.83$. Monomer/water $(\mathrm{v} / \mathrm{v})=0.03 / 1$ (open circles), 0.1/1 (closed circles).
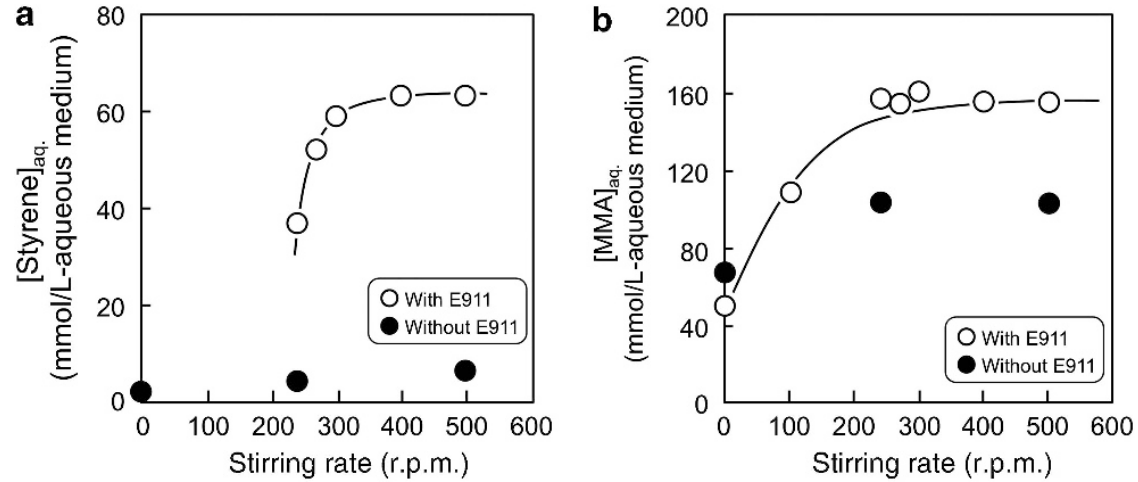

Figure 3 Monomer (styrene (a) and MMA (b)) concentrations in the aqueous phase (water and micelles) for 100 min at $70^{\circ} \mathrm{C}$ before starting the emulsion polymerization with (open circles) and without (closed circles) E911 as a function of the stirring rate: monomer $2.0 \mathrm{~g}$; E911 $0.20 \mathrm{~g}$; water $60 \mathrm{~g}$. Initial monomer concentration (mmoll-1-water): styrene, 320; MMA, 332.

difference in the amounts of the monomer phases, because E911 concentrations in the monomer phases in both systems were the same. After stirring at 500 r.p.m. for $100 \mathrm{~min}$ at a temperature of $70{ }^{\circ} \mathrm{C}$, the amount of the MMA phase was only $\sim 30 \%$ of the styrene phase because MMA is more soluble in water than is styrene.

When emulsion polymerization at $10 \mathrm{wt} \%$ MMA concentration was carried out at 240 or 500 r.p.m. (Figure 2b), [E911 $]_{\text {aq. }}$ decreased with the increase in the stirring rate, similarly to styrene (Figure 2a). E911 preferentially existed in the MMA phase rather than in the aqueous phase, because the polymerization temperature is close to the cloud point of E911.

\section{Monomer concentration in the aqueous phase just before the} emulsion polymerization of styrene or MMA

Figure 3 shows monomer (styrene and MMA) concentrations $\left([\text { Styrene }]_{\mathrm{aq}}\right.$. and $[\mathrm{MMA}]_{\mathrm{aq}}$ ) in the aqueous media (water and micelles) just before starting the emulsion polymerization for $100 \mathrm{~min}$ at $70^{\circ} \mathrm{C}$ as a function of the stirring rate. $[\mathrm{MMA}]_{\mathrm{aq}}$. as well as $[\text { Styrene }]_{\text {aq. }}$ increased to a plateau value $\left(157 \mathrm{mmoll}^{-1}\right)$ with the increase in the stirring rate, although the plateau concentration was 2.5 times higher in MMA than in styrene. The MMA (M)-240 system reached a plateau value (equilibrium state), but the styrene (S)-240 system failed to reach an equilibrium state. These results indicate that the MMA transferred more rapidly than styrene to the aqueous medium because of the higher solubility of MMA in water.
The difference of $[\text { monomer }]_{\text {aq. }}$ with $(\bigcirc) /$ without $(\bigcirc)$ E911 indicates that the numbers of monomers solubilized in E911 micelles and those in M-240 and M-500 systems were nearly equal to the monomers solubilized in the S-500 system, which was almost $60 \mathrm{mmoll}^{-1}$-water.

Figure 4 shows the number-average diameters $\left(d_{\mathrm{n}}\right)$ of micelles in S-500, S-240, M-240 and M-500 systems just after stirring for $100 \mathrm{~min}$ at $70{ }^{\circ} \mathrm{C}$ in the absence of KPS. The $d_{\mathrm{n}}$ values of E911 micelles in S-240 (a) and S-500 (b) systems were $8.3 \pm 1.7$ and $40 \pm 5.7 \mathrm{~nm}$, respectively. As the $d_{\mathrm{n}}$ of free E911 micelles was $2.1 \pm 0.6 \mathrm{~nm}$, the E911 micelles in the S-240 system were swollen with styrene, but the swelling was remarkable in the S-500 system. The difference can reasonably be explained from the values of $[\mathrm{E} 911]_{\mathrm{aq}}$ and $[\text { styrene }]_{\mathrm{aq}}$. (Figures 2, 3a. The $d_{\mathrm{n}}$ values of E911 micelles in M-240 and M-500 systems were $11.1 \pm 2.8$ and $16.0 \pm 3.5 \mathrm{~nm}$, respectively. The $d_{\mathrm{n}}$ values were much smaller than that in the S-500 system, because the numbers of E911 micelles in the aqueous media were larger than that in the S-500 system. The total amounts of monomer solubilized in the total E911 micelles existing in the aqueous media were almost the same ( $\sim 60 \mathrm{mmoll}^{-1}$-water) in M-240, M-500 and S-500 systems (see Figures 2, 3), resulting in less monomer solubilized in individual micelles in the M-240 and M-500 systems than in the S-500 system.

The difference in monomer polarity, as well as the stirring, significantly affected the partitioning of monomer and E911 just before starting emulsion polymerization. 
Emulsion polymerization of styrene and MMA with E911

Figure 5 shows conversion-time plots for emulsion polymerizations in the S-240 (O), S-500 (○) (a) and M-240 ( ○), M-500 (○) (b) systems. The polymerization of MMA proceeded faster than the polymerization of styrene, and the stirring rate had little effect on the polymerization rate in both monomer systems.

Figure 6 shows TEM photographs of PS and PMMA particles prepared by emulsion polymerization at the stirring rates of 240 and 500 r.p.m. For styrene, the particles prepared at both stirring rates were quite different in size and morphology. Only nano-sized $\left(d_{\mathrm{n}}=70 \mathrm{~nm}\right)$ spherical particles were prepared at 240 r.p.m., whereas at 500 r.p.m., micron-sized $\left(d_{\mathrm{n}} \approx 1 \mu \mathrm{m}\right)$ 'bur-like' nonspherical particles and submicron-sized $\left(d_{\mathrm{n}} \approx 100 \mathrm{~nm}\right)$ spherical particles were prepared. In the case of MMA, ca. 250-nm-sized, almost spherical particles, with surface roughness much lower than those in the S-500 system, were prepared at both stirring rates.
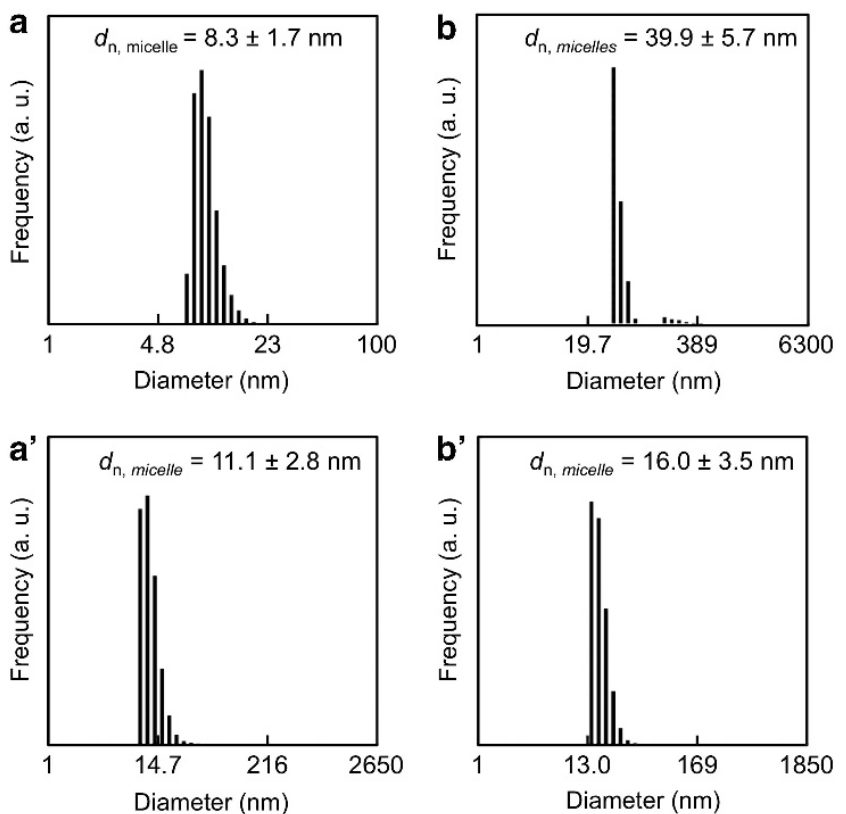

Figure 4 Number-average diameter distributions of monomer (styrene (a, b) and MMA $\left(\mathbf{a}^{\prime}, \mathbf{b}^{\prime}\right)$ ) emulsions stirred at $240\left(\mathbf{a}, \mathbf{a}^{\prime}\right)$ and $500\left(\mathbf{b}, \mathbf{b}^{\prime}\right)$ r.p.m. for $100 \mathrm{~min}$ at $70^{\circ} \mathrm{C}$. Free micelles $(\mathrm{nm}): d_{\mathrm{n}}=2.1 \pm 0.6 ; d_{\mathrm{w}}=3.0 \pm 1.6$.
Table 2 shows the amounts of E911 incorporated inside the PS and PMMA particles. Weight percentage of incorporated E911 relative to the total amount fed was $5 \mathrm{wt} \%$ in the $\mathrm{S}-240$ system but above $70 \mathrm{wt} \%$ ( $7.0 \mathrm{wt} \%$ relative to PS) in the S-500 system. In the M-240 and M-500 systems, those values were 72 and $65 \mathrm{wt} \%$, similar to that of the S-500 system. This tendency was similar with the partitioning states of monomer and emulsifier just before starting emulsion polymerizations, as described above. The glass transition temperature of PS particles with incorporated E911 (5.5 wt\% in particles) after removing E911 adsorbed on the particles by repeated washing was $\sim 10^{\circ} \mathrm{C}$ lower than for the particles without incorporated E911 [15].

The partitioning of E911 and monomer before starting polymerization strongly affected the particle formation and the incorporation of E911. To clarify the influences, the polymerization behaviors were observed in detail.

Figure 7 shows the variations in the monomer concentrations in the particles during emulsion polymerizations of S-240, S-500, M-240 and M-500 systems. In the S-500, M-240 and M-500 systems, the polymerizations proceeded with high monomer concentrations in the particles based on the high interfacial area of styrene droplets and the high solubility of MMA in water. In these systems, E911 transferred from droplets into the particles via the aqueous media together with monomer throughout the polymerizations, resulting in a large amount of E911 incorporated inside the particles (see Table 2).

Figures 8 and 9 show TEM photographs of PS particles during the emulsion polymerization of styrene at 240 and 500 r.p.m. Although the polymerizations proceeded similarly (as shown in Figure $5 \mathrm{a}$ ), the particle formations were clearly different. In the S-240 system (Figure 8), primary particles were formed at the initial stage of the polymerization and gradually grew throughout the polymerization. New particles were also observed during the polymerization, and these particles were stable because of high $[\mathrm{E} 911]_{\mathrm{aq}}$. This stability suggests that mainly micellar nucleation occurred. In the S-500 system, primary particles aggregated and formed large particles at 26 and 30\% conversions; new particles were also formed during the polymerization (Figure 9). The main particles grew and adsorbed numerous new small particles that were unstable because insufficient $[\text { E911 }]_{\text {aq. }}$ was available for coverage of the surfaces of the small particles due to the partitioning of E911 to the monomer phase, possibly resulting in the 'bur-like' nonspherical particles. To clarify the situation, comparison of the molecular weights and distributions of polymer particles (S-240 and S-500) with smaller and larger particles was necessary. The number of the free smaller particles was
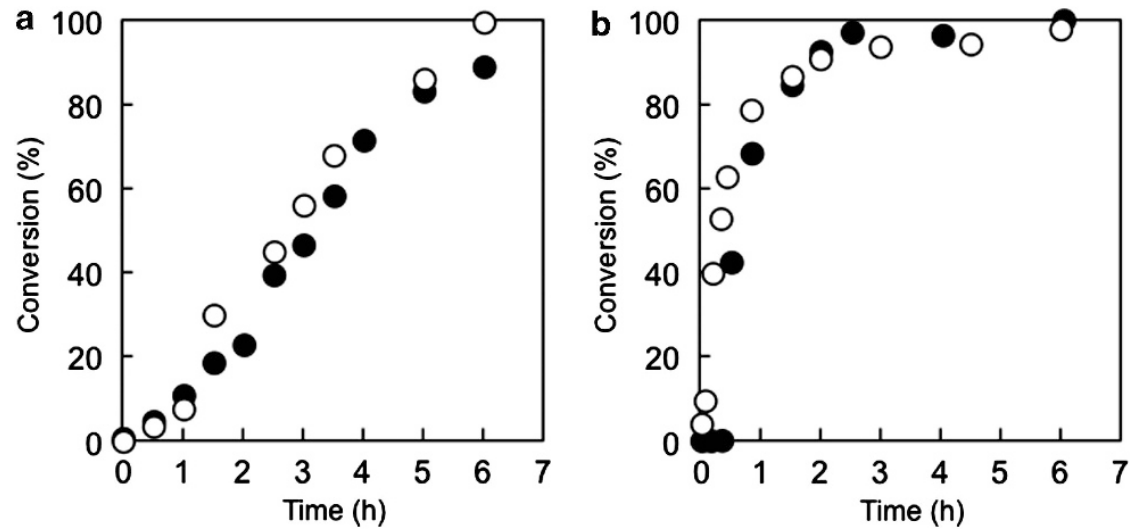

Figure 5 Conversion-time plots for emulsion polymerizations of styrene (a) and MMA (b) at 240 (open circles) and 500 (closed circles) r.p.m. 

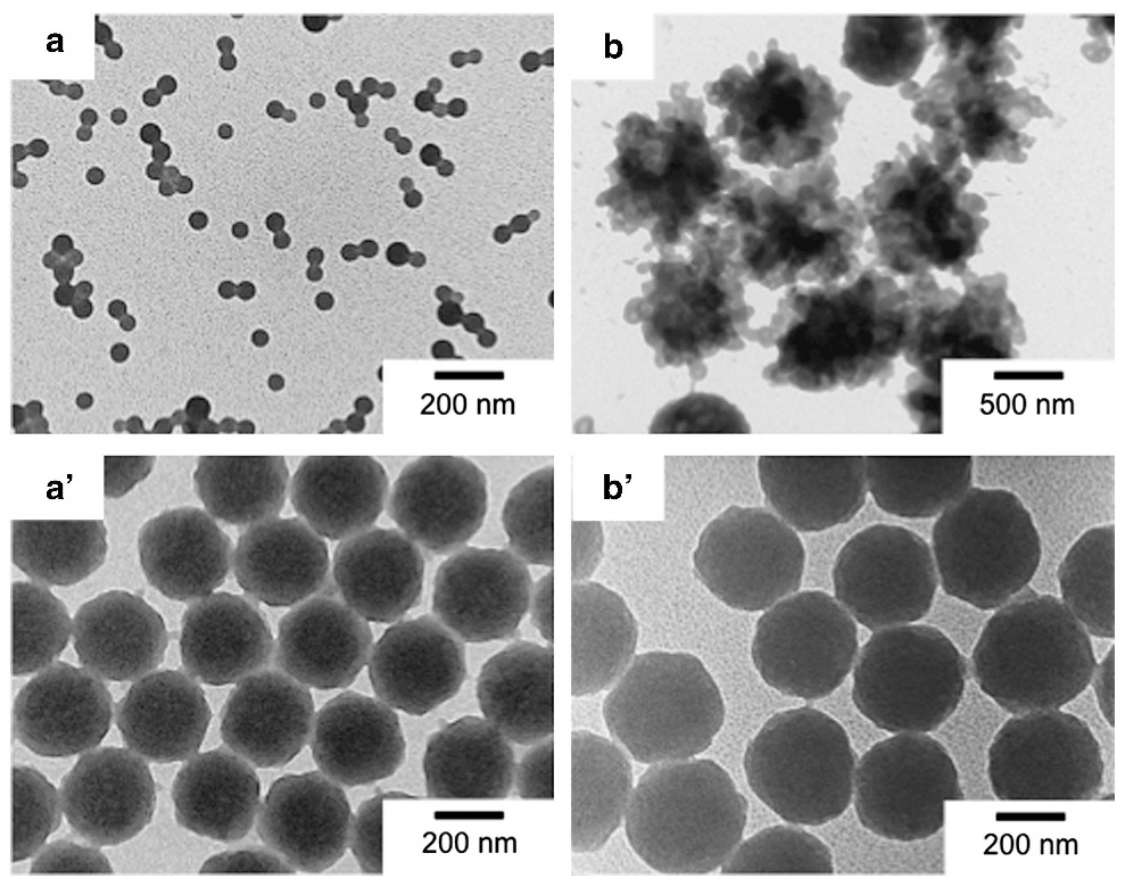

Figure 6 TEM micrographs of PS (a, b) and PMMA ( $\left.\mathbf{a}^{\prime}, \mathbf{b}^{\prime}\right)$ particles prepared by emulsion polymerization at $240\left(\mathbf{a}, \mathbf{a}^{\prime}\right)$ and $500\left(\mathbf{b}, \mathbf{b}^{\prime}\right)$ r.p.m.
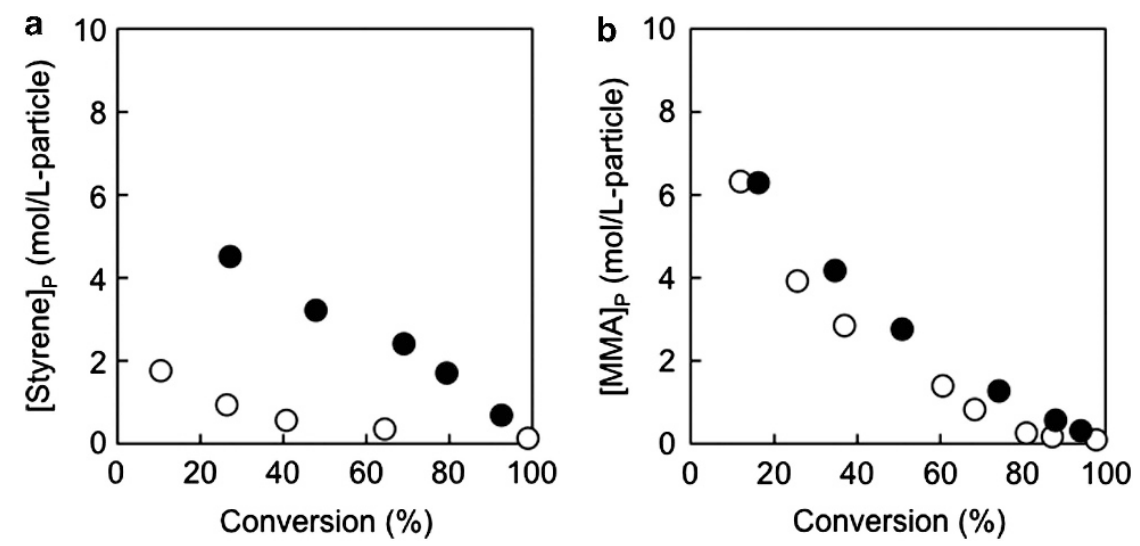

Figure 7 Styrene (a) and MMA (b) concentrations in the particle as functions of total conversion in the emulsion polymerizations of styrene and MMA at stirring rates of 240 (open circles) and 500 (closed circles) r.p.m.

Table 2 Weight percentages of incorporated E911 inside particles prepared by emulsion polymerization in the S-240, S-500, M-240 and M-500 systems

\begin{tabular}{lcccc}
\hline & $S-240$ & $S-500$ & $M-240$ & $M-500$ \\
\hline Incorporation $^{\mathrm{a}}(\%)$ & $5.0(0.50)$ & $70(7.0)$ & $72(7.2)$ & $65(6.5)$ \\
\hline
\end{tabular}

Abbreviation: E911, emulgen 911.

aBased on total weight of emulsifier (weight of polymer); measured by GPC.

too few to measure them with GPC. As shown in Figures 9b, $\mathrm{c}$ and d, in the middle stage (30-69\% conversion), PS particles had domains with low contrast (less electron density) originating from the hollow structure inside. This point will be reported in detail in a future paper.

Figures 10 and 11 show TEM photographs of PMMA particles prepared during the emulsion polymerization in $\mathrm{M}-240$ and $\mathrm{M}-500$ systems. In both systems, many primary particles $\left(d_{\mathrm{n}}=20 \mathrm{~nm}\right)$ were instantly formed, as reflected by the states before starting emulsion polymerization. The aggregation of the primary particles resulted in ca. 250-nm-sized particles in the initial stage of the polymerization until $\sim 10 \%$ conversion. Most of the E911 adsorbed on the primary particles seems to be incorporated on the inside of the $\sim 250$-nm-sized particles. Throughout the polymerization, new particles other than the 

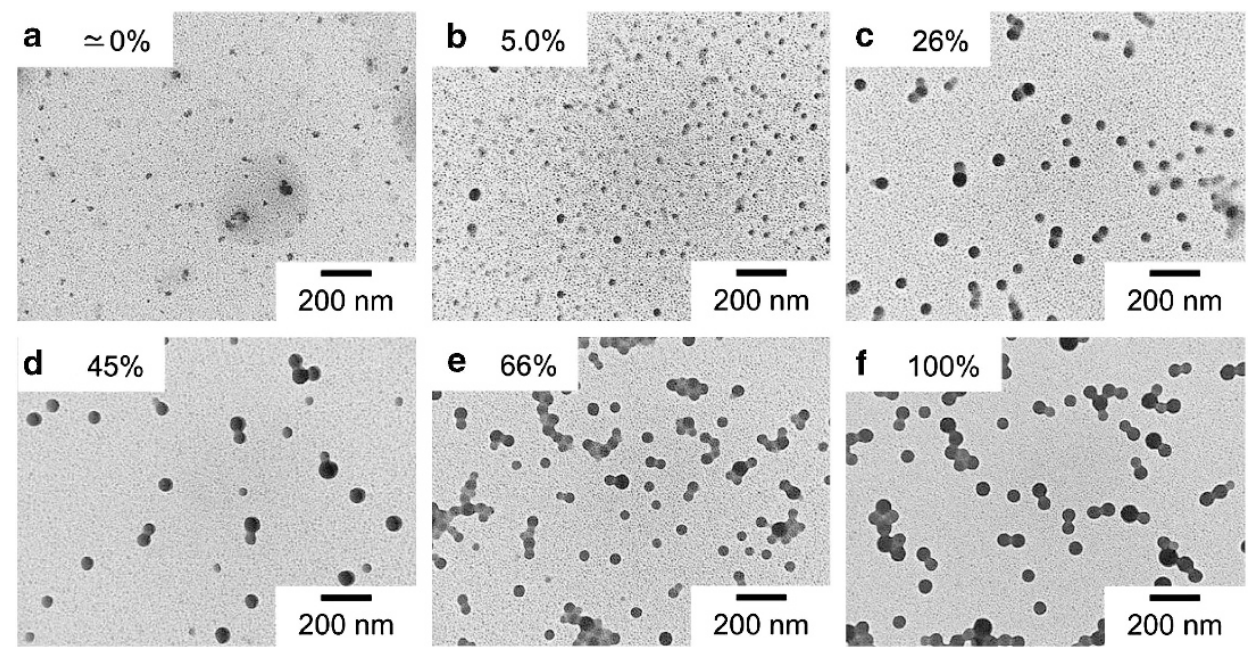

Figure 8 TEM micrographs of PS particles prepared by emulsion polymerization at 240 r.p.m. under the conditions listed in Table 1 . Percentages on the images indicate conversions.
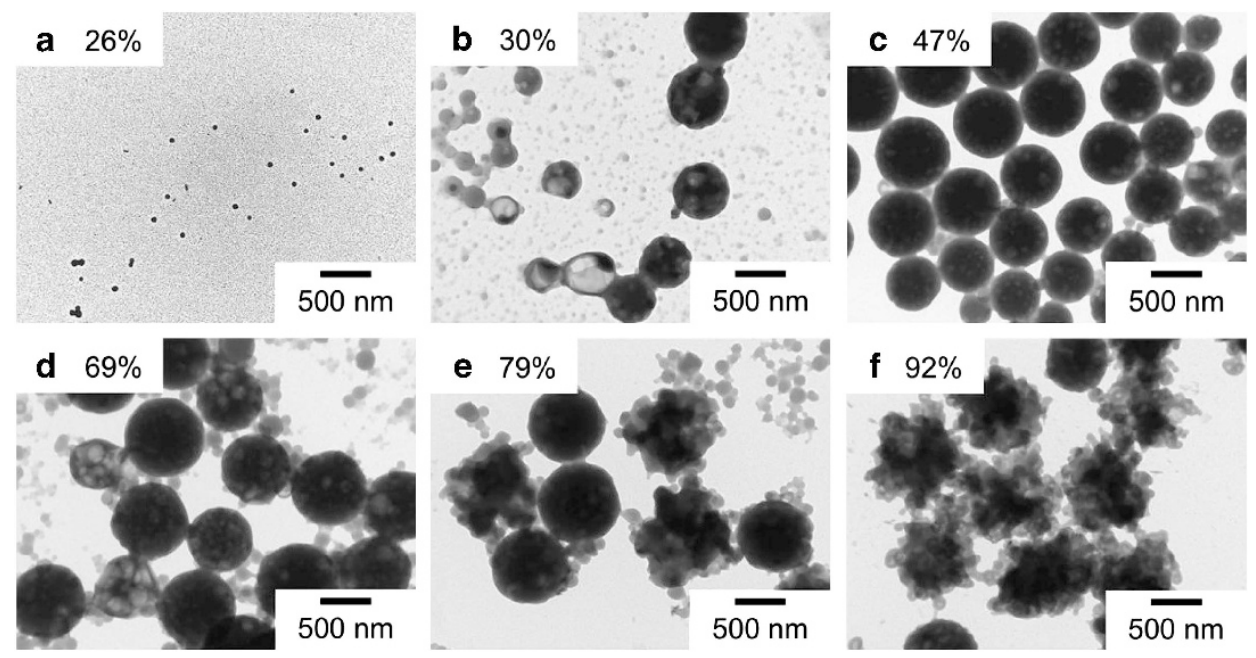

Figure 9 TEM micrographs of PS particles prepared by emulsion polymerization at 500 r.p.m. under the conditions listed in Table 1 . Percentages on the images indicate conversions.

250-nm-sized particles were not observed, also confirmed by DLS results. These results suggest that all MMA oligomer radicals generated in aqueous media entered particles swollen with monomers; thus, the polymerizations proceeded without the formation of new particles.

The above results demonstrate that monomer polarity strongly affected the partitioning of monomer and emulsifier in an aqueous phase before starting emulsion polymerization, resulting in differences in particle formation and in the incorporation of the nonionic emulsifier inside polymer particles.

\section{CONCLUSIONS}

The effects of monomer polarity on the partitioning of monomer and emulsifier before starting emulsion polymerization with nonionic emulsifier were investigated. Particle formation and incorporation inside polymer particles were also investigated. Partitioning of the monomer from a monomer to an aqueous phase and partitioning of the emulsifier from the aqueous phase to the monomer phase was strongly affected by the monomer polarity just before starting emulsion polymerization. Although the S-500 system nearly reached the equilibrium state, the S-240 system was in the nonequilibrium state. However, in the case of MMA, the stirring rate made little difference in the condition of the monomer solution. MMA was apparently transferred from the monomer phase to the aqueous phase faster than styrene because of higher MMA monomer polarity. The resulting PMMA particles also showed a similar morphology regardless of the stirring rate, and assumed the size of $\sim 250$-nm-sized nonspherical particles. However, the PS particles showed different morphologies in S-240 and S-500 systems. E911 was incorporated inside PMMA particles at $\sim 70 \mathrm{wt} \%$ in the $\mathrm{M}-240$ and $\mathrm{M}-500$ systems, nearly the same value as in the S-500 system. These results indicate that monomer polarity strongly influenced the partitioning of monomer and emulsifier before starting the emulsion polymerization, resulting in the differences in particle formation and the incorporation of the nonionic emulsifier inside the polymer particles. 

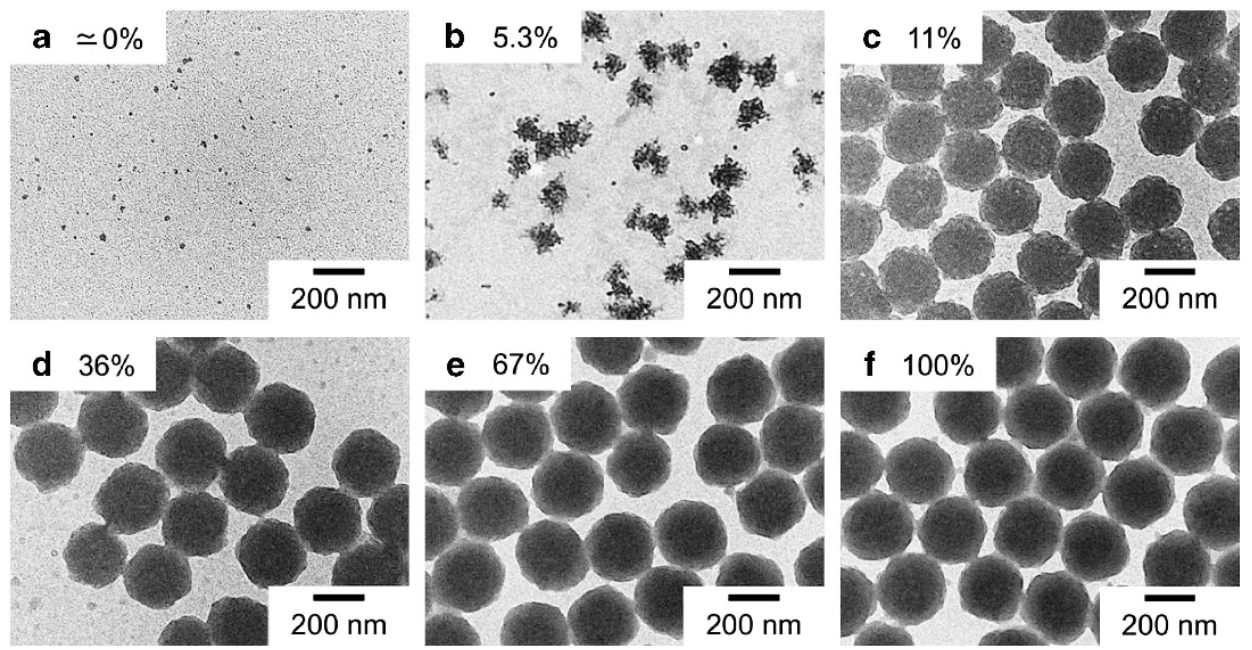

Figure 10 TEM micrographs of PMMA particles prepared by emulsion polymerization at 240 r.p.m. under the conditions listed in Table 1 . Percentages on the images indicate conversions.
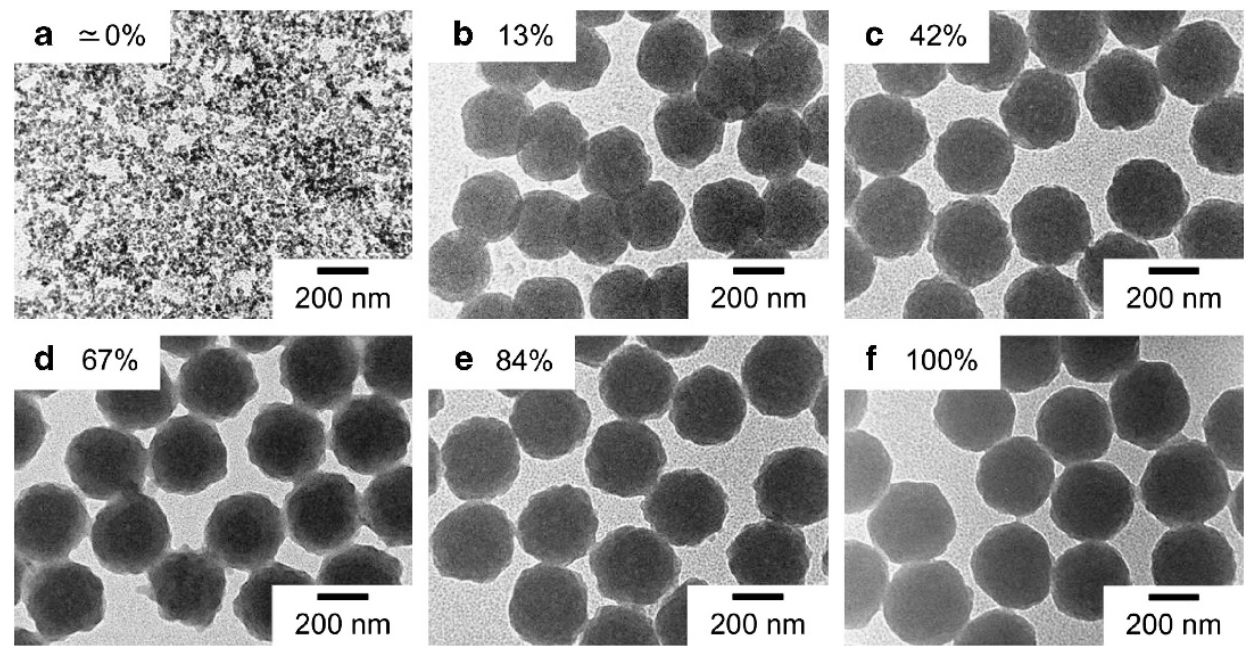

Figure 11 TEM micrographs of PMMA particles prepared by emulsion polymerization at 500 r.p.m. under the conditions listed in Table 1 . Percentages on the images indicate conversions.

\section{ACKNOWLEDGEMENTS}

This work was supported by a Grant-in-Aid for Scientific Research (A) (Grant 21245050) from the Japan Society for the Promotion of Science (JSPS).

1 Piirma, I. \& Chang, M. Emulsion Polymerization of Styrene: Nucleation Studies with Nonionic Emulsifier. J. Polym. Sci. Polym. Chem. Ed. 20, 489-498 (1982).

2 Piirma, I. \& Maw, T. S. Emulsion polymerization: some nonionic emulsifier effects Polym. Bull. 11, 497-504 (1984).

3 Ozdeger, E., Suldol, E. D., El-Aasser, M. S. \& Klein, A. Role of the Nonionic Surfactant Triton X-405 in Emulsion Polymerization. I. Homopolymerization of Styrene. J. Polym. Sci. A Polym. Chem. 35, 3813-2825 (1997).

4 Ozdeger, E., Suldol, E. D., El-Aasser, M. S. \& Klein, A. Role of the Nonionic Surfactant Triton X-405 in Emulsion Polymerization. II. Homopolymerization of $n$-Butyl Acrylate. J. Polym. Sci. A Polym. Chem. 35, 3827-3835 (1997).

5 Ozdeger, E., Suldol, E. D., El-Aasser, M. S. \& Klein, A. Role of the Nonionic Surfactant Triton X-405 in Emulsion Polymerization. III. Copolymerization of Styrene and $n$-Butyl Acrylate. J. Polym. Sci. A Polym. Chem. 35, 3837-3846 (1997).

6 Capek, I. \& Chudej, J. On the fine emulsion polymerization of styrene with non-ionic emulsifier. Polym. Bull. 43, 417-424 (1999).
7 Lin, S. Y., Capek, I., Hsu, T. J. \& Chern, C. S. On the emulsion polymerization of styrene in the presence of a noninonic emulsifier. J. Polym. Sci. A Polym. Chem. 37, 4422-4431 (1999).

8 Okubo, M., Furukawa, Y., Shiba, K. \& Matoba, T. Inclusion of nonionic emulsifier inside polymer particles produced by emulsion polymerization. Colloid Polym. Sci. 281, 182-186 (2003).

9 Okubo, M., Kobayashi, H., Matoba, T. \& Oshima, Y. Incorporation of nonionic emulsifier inside particles in emulsion polymerization: Mechanism and methods of suppression. Langmuir 22, 8727-8731 (2006).

10 Chaiyasat, A., Kobayashi, H. \& Okubo, M. Incorporation of nonionic emulsifier inside methacrylic polymer particles in emulsion polymerization. Colloid Polym. Sci. 285, 557-562 (2007).

11 Okada, M., Matoba, T \& Okubo, M. Influence of nonionic emulsifier included inside carboxylated polymer particles on the formation of multihollow structure by the alkali/ cooling method. Colloid Polym. Sci. 282, 193-197 (2003).

12 Kobayashi, H., Miyanaga, E. \& Okubo, M. Preparation of hollow polymer particles with a single hole in the shell by SaPSeP. Langmuir 23, 8703-8708 (2007).

13 Okubo, M., Chaiyasat, A., Yamada, M., Suzuki, T. \& Kobayashi, H. Incorporation of nonionic emulsifiers inside styrene-methacrylic acid copolymer particles during emulsion polymerization. Colloid Polym. Sci. 285, 1755-1761 (2007).

14 Matsusaka, N., Suzuki, T. \& Okubo, M. Colloid Polym. Sci. (in press, 2012). 